JOURNAL DE PHYSIQUE IV

Colloque C2, supplément au Journal de Physique III, Volume 5, février 1995

\title{
Magnetic Aspects of Martensitic Transformations in FeNi Alloys
}

M. Acet, T. Schneider and E.F. Wassermann

Tieftemperaturphysik, Gerhard-Mercator-Universität, 47048 Duisburg, Germany

\begin{abstract}
Fe}_{100-\mathrm{x}} \mathrm{Ni}_{\mathrm{x}}$ alloys for $\mathrm{x}<34$ at\% i.e., up to the structurally stable Invar concentration, undergo a martensitic transformation. In the parent fcc-phase these alloys show a volume enhancement which is known as the anti-Invar effect. This effect disappears at a concentration at which the martensitic transformation also no longer takes place. In a narrow concentration range of about $30 \leq x<34$ at\% Invar, anti-Invar and the martensitic transformation can be observed simultaneously. The Invar and the anti-Invar effects are understood to be due to the instability of the magnetic moment with respect to a volume change, i.e. the moment-volume instability. Using thermal expansion measurements on $\mathrm{Fe}_{100-x} \mathrm{Ni}_{x}$, with $0 \leq x \leq 37$ at \%, we determine critical volumes of the $\gamma$ phase at the martensitic transformations. Furthermore we show the existence of Invar behavior in the rest Austenite in $\mathrm{Fe}_{70} \mathrm{Ni}_{30}$.
\end{abstract}

\section{INTRODUCTION}

The $\alpha-\gamma$ (bcc-fcc) transformation in Fe at $\mathrm{T}=1184 \mathrm{~K}$ ( $\mathrm{A}_{3}$ point $)$ has been the subject of intensive research in metallurgy and physics. One of the open questions related to the transformation is why the atomic volume of the low temperature product $\alpha$-phase is larger than that of the high temperature parent $\gamma$-phase at the $A_{3}$ point. In other words, what internal effect causes the atomic volume $\mathrm{V}_{\mathrm{a}}$ to increase when going through the transition with decreasing temperature. The answer to this question clearly lies in the understanding of the microscopic mechanism of the transformation. The formalism of the mechanism should certainly take into account the magnetic interactions in $\alpha$ and $\gamma-\mathrm{Fe}$, since a comparison of the relative differences in the Gibbs free energies, $\Delta \mathrm{G}$, of the $\alpha, \gamma$ and hcp $\varepsilon$ phases (Fig. 1) show that without ferromagnetism in the $\alpha$-phase the paramagnetic (PM) $\gamma$-phase would have been stable down to about $500 \mathrm{~K}$ [1-

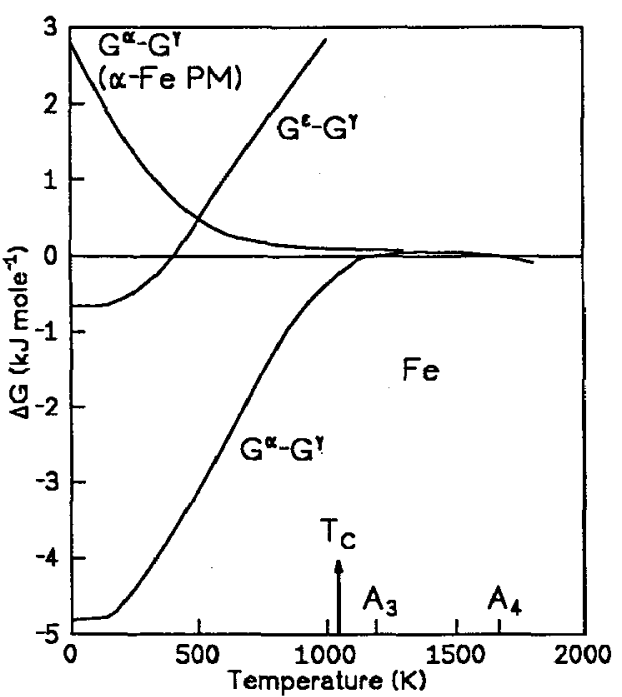

Fig. 1. Difference in the Gibbs free energies of the $\alpha$, $\gamma$ and $\varepsilon$-phases in Fe.

$3]$. Below this temperature the $\varepsilon$-phase would have been stable. This very basic fact demonstrates clearly how magnetism, and therefore electronic properties, can play a vital rôle in the stabilization of a 
particular structure. However, magnetic ordering is not necessarily an essential factor. Rather, as in Fe, magnetic st. st range correlations above the Curie temperature, $T_{C}=1043 \mathrm{~K}$, can be also sufficiently strong to contribute to the stabilization of the $\alpha$-phase.

$\gamma-\mathrm{Fe}$ is stable between the $A_{3}$ and $A_{4}$ points $(1184<T<1665 \mathrm{~K}$ ). It is an anti-Invar in which large fluctuations in the magnetic moment and volume (moment-volume instability) give rise to an enhanced anharmonicity leading to an excess thermal expansion. [4-6] It is antiferromagnetic below the Néel temperature, $T_{N}=70 \mathrm{~K}$, with a magnetic moment $\mu=0.5 \mu_{\mathrm{B}}$ but, in the stability range predominantly short range ferromagnetic (FM) correlations with $\mu=1.2 \mu_{B}$ are known to exist from neutron diffraction experiments. [7] In the ground state it has been shown by magnetic investigations of artificial fcc- $\mathrm{Fe}$ lattices that $\gamma-\mathrm{Fe}$ is AF below a critical volume $V_{c}=12.05 \times 10^{-3} \mathrm{~nm}^{3}$ and $F M$ above it [8]. Therefore, just as FM short range correlations contribute to the stabilization of the $\alpha$-phase of Fe, one might expect the FM short range correlations in $\gamma-\mathrm{Fe}$ to provide the internal force to drive the structure to the larger volume $\alpha$-phase on decreasing temperature.

Addition of $\mathrm{Ni}$ to $\mathrm{Fe}$ stabilizes the $\gamma$-phase to lower temperatures. In Fe-rich FeNi alloys the $\alpha-\gamma$ transformation takes the form of a Martensitic transformation characterized by a diffusionless process. In the concentration range where martensitic transformations take place, i.e., up to about 34 at $\% \mathrm{Ni}$, the antiInvar effect is also observed [9]. Anti-Invar behavior and Martensite disappear almost together near this concentration, after which Invar properties set in. Therefore, in order to begin a systematic investigation of the magnetic aspects of the $\alpha-\gamma$ transformation in Fe based alloys, we examine the properties of the transformation in the FeNi system. In the present paper we study the volumetric properties of $\mathrm{FeNi}$ alloys and discuss its presence in Invar and anti-Invar alloys.

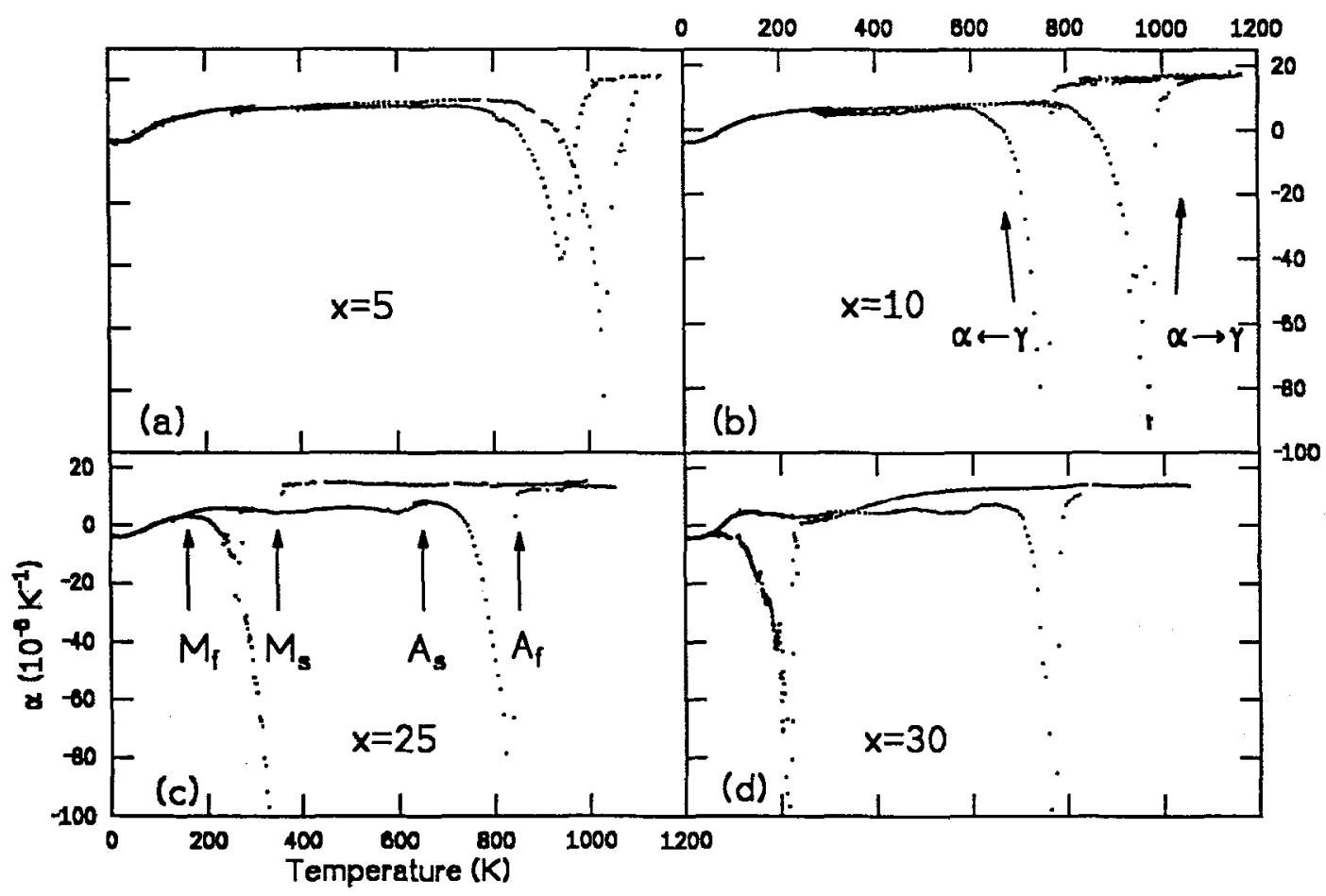

Fig. 2. $\alpha(\mathrm{T})$ of $\mathrm{Fe}_{100-\mathrm{x}} \mathrm{Ni}_{\mathrm{x}}$. Measurement directions are indicated in part (b). The transformation temperatures are identified as in part $(\mathrm{c})$. 


\section{EXPERIMENT}

The thermal expansion $\Delta \mathrm{l} / \mathrm{h}$ of five $\mathrm{Fe}_{100-\mathrm{x}} \mathrm{Ni}_{\mathrm{x}}$ alloys with concentrations $\mathrm{x}=5,10,25,30$ and 35 at $\%$ was measured in two separate capacitance dilatometers functioning in the ranges $4<\mathrm{T}<300 \mathrm{~K}$ and $300<\mathrm{T}<1200 \mathrm{~K}$ respectively. $\Delta \mathrm{l} / \mathrm{l}$ were measured first in the low temperature apparatus on decreasing temperature and then on increasing temperature. They were then transfered to the high temperature apparatus and measured on increasing temperature through the $A_{f}$ temperature, and finally back to room temperature. Data were taken on equilibrium roughly $3 \mathrm{~K}$ apart in the low temperature dilatometer and about $5 \mathrm{~K}$ apart in the high temperature dilatometer.

\section{RESULTS}

Fig. 2 shows $\alpha(T)$ for the alloys that undergo a Martensitic transformation. The Martensitc and Austenitic transformation temperatures $\left(M_{s}, M_{f}\right.$ and $\left.A_{s}, A_{f}\right)$ are identified as shown in Fig $2(c)$. ' $s$ ' and ' $f$ denote 'start' and 'final' respectively. The direction of temperature change is indicated in Fig. 2(b). On coming down from high temperature the samples go through $\mathrm{M}_{\mathrm{s}}$ below which they are essentially $\alpha$ but also contain rest Austenite. The amount of rest Austenite in the $x=10$ and $x=5$ at $\%$ alloys at low temperatures is negligeble [10]. In all measurements $\alpha(T)$ of the $\gamma$-phase at high temperatures is somewhat larger than in the Martensite phase, in accordance with the denser packed structure of the $\gamma$ phase. For the $\mathrm{x}=30$ at $\%$ alloy the decreasing temperature data within the $\gamma$-phase shows a drop before the $\mathrm{M}_{\mathrm{s}}$ temperature is reached. This drop is associated with the Invar effect, which is known to occur in this alloy [9].

\subsection{The Magnetic and Structural Phase Diagram}

The phase diagram showing the structural and magnetic transformation temperatures is plotted in Fig. 3. The subscripts 's' and ' $\mathrm{f}$ ' refer to start and final, respectively. $\mathrm{T}_{\mathrm{N}}$ begins at $70 \mathrm{~K}$ for $\gamma-\mathrm{Fe}$ and is expected to gradually decrease to $0 \mathrm{~K}$ near $\mathrm{x}=20-25$ at $\%$ where ferromagnetic ordering sets in, as denoted by the $T_{C}$ line. The present $\alpha(T)$ data show that the separations between the $M_{s}$ and $M_{f}$ lines and the $A_{s}$ and $A_{f}$ lines are somewhat larger than earlier reported [10]. In a narrow concentration range around $x=30$ at\% the Martensitic transformation occurs below $T_{C}$, i.e. the parent phase is magnetically ordered. For other concentrations the parent phase is PM. Tab. I summarizes the state of magnetic ordering in the $\alpha$ and $\gamma$-phases at $M_{s}$. The possible combinations of FM and PM in the two phases show that magnetic ordering is not essential for the Martensitic transformation in FeNi alloys.

Tab. 1. Magnetic ordering of the $\alpha$ and $\gamma$-phases of $\mathrm{Fe}_{100-\mathrm{x}} \mathrm{Ni}_{\mathrm{x}}$ at $\mathrm{M}_{\mathrm{s}}$

\begin{tabular}{|c|c|c|}
\hline $\mathrm{x}(\mathrm{at} \%)$ & parent $(\gamma)$ & product $(\alpha)$ \\
\hline 0 & PM & PM \\
\hline 5 & PM & PM \\
\hline 10 & PM & FM \\
\hline 25 & PM & FM \\
\hline 30 & FM & FM \\
\hline
\end{tabular}

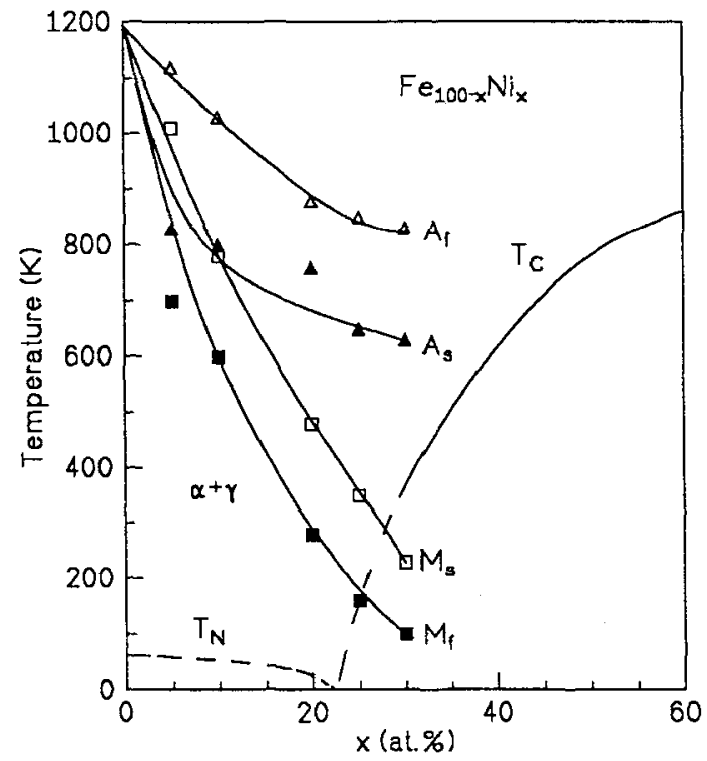

Fig. 3. The phase diagram of $\mathrm{Fe}_{100-x} \mathrm{Ni}_{\mathrm{X}}$ showing $\mathrm{T}_{C}$ and $\mathrm{T}_{\mathrm{N}}$ for the fec phase and structural transformation temperatures. 


\subsection{Low Temperature $\alpha(T)$}

Fig. 4 shows the low temperature portion $(4<T<400 \mathrm{~K})$ of the $\alpha(T)$ data obtained on increasing temperature. Due to adequate temperature stability for $4<T<300 \mathrm{~K}, \alpha(\mathrm{T})$ of these curves could be calculated as the slope of consecutive $\Delta \mathrm{l} / \mathrm{l}$ data. The curves for $\mathrm{x}=30$ and $x=25$ at $\%$ show an interesting development with increasing $\mathrm{Ni}$ concentration. The almost completely $\alpha$-phase $x=5$ and $x=10$ at $\%$ alloys show regular behavior in $\alpha(T)$ (the curve for $x=10$ at\% is not shown for clarity). As the $\mathrm{Fe}$ concentration decreases a hump develops with $x=25 a t \%$, and becomes more distinct for $x=30$ at $\%$. At the same time the higher temperature ends of the data tend to level off. When these two data are compared to the pure austenite $x=35$ or $x=37$ at $\%$ Invar alloy, one sees that the features in $\alpha(T)$ of the $x=30$ at $\%$ alloy resembles that of Invar. This means that rest austenite in the $x=30$ at $\%$ alloy has sufficient influence to exhibit Invar properties in the mixed $\alpha+\gamma$ phase. Therefore, we show clearly that rest Austenite in $\mathrm{FeNi}$ alloys which undergo martensitic transformations and at the same time lie close to the Invar composition exhibit coexisting Invar and Martensite properties

\section{DISCUSSION}

It is a general fact that fcc-alloys of $\mathrm{Fe}$ undergoing Martensitic transformations experience an increase in $V_{a}$ when going through $M_{s}$ with decreasing temperature. That FM short range correlations in the $\alpha$-phase are responsible for the stabilization of the bcc structure is a fact established from thermodynamical analysis of $\mathrm{Fe}$. A similar interplay between magnetism and structure can exist in the $\gamma$-phase as well. Namely, the strength of the magnetic short range correlations can increase with decreasing temperature due to decrease of lattice vibration amplitude. At the same time the volume contracts due to decrease of temperature until the volume can no longer support the fcc structure. Thereafter and a phase transformation to larger volume bcc state will be triggered. Unfortunately, to verify this idea a detailed complimentary study of the size magnetic moment of the correlations in these alloys is required. Such a study has not been undertaken until now. Nevertheless, we plot in Fig. $5 \mathrm{~V}_{\mathrm{a}}$ of the $\gamma$-phase at $\mathrm{M}_{\mathrm{s}}$, which corresponds to the smallest volume that the $\gamma$-phase can acquire before the fcc structure becomes unstable. The increase in $V_{a}$ above $x=25$ at $\%$ is due to the Invar effect. The data for $x=35$ at $\%$ Invar is obtained

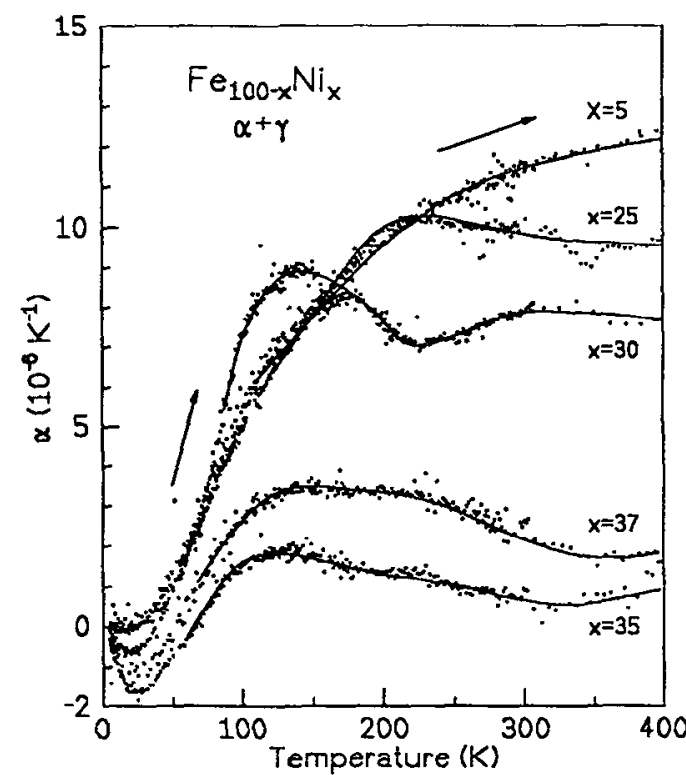

Fig. 4. $\alpha(\mathrm{T})$ of $\mathrm{Fe}_{100-\mathrm{x}} \mathrm{Ni}_{\mathrm{x}}$ in the $\alpha-\gamma$ mixed state. Data taken on increasing temperature after being cooled to $T=4 \mathrm{~K}$.

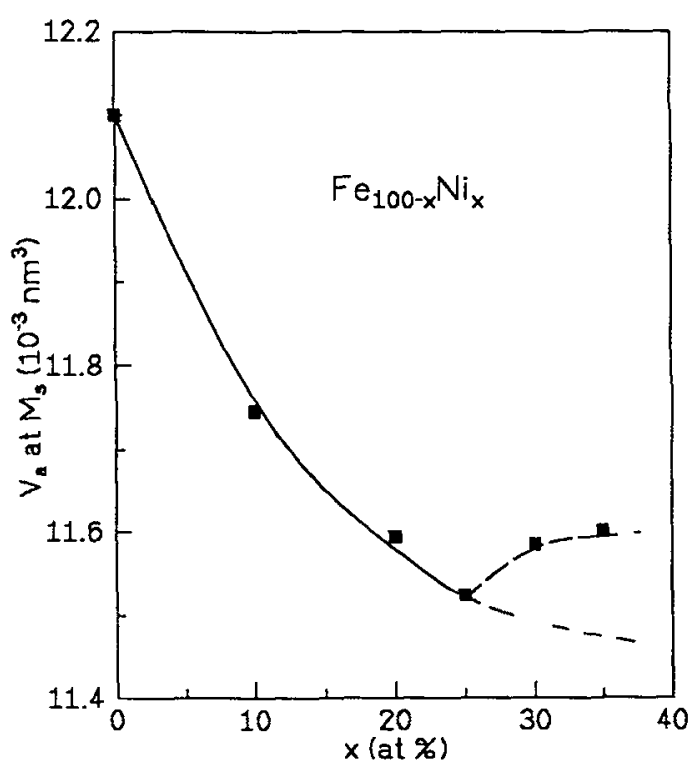

Fig. 5. The fcc $V_{a}$ at the $M_{s}$ temperature of $F e_{100-x} N_{x}$ 
for $T \rightarrow 0$ since for this concentration $M_{s}=0 \mathrm{~K}$. A further study of the size of the paramagnetic moment can lead to a relationship between magnetic moment and volume at $M_{s}$. This relationship can lead to a critical condition for the transformation.

Anti-Invar is a property of elemental $\mathrm{Fe}$ and this property is carried into $\mathrm{Fe}_{\mathbf{x}} \mathrm{Ni}_{100-\mathrm{x}}$ up to about $\mathrm{x}=30 \mathrm{at} \%$. As long as the temperature of these alloys is high enough, so that FM short range correlations in the $\gamma$-phase predominate as in $\gamma$-Fe, Martensitic transformations can take place. Therefore the antiInvar property is not likely to have a dominating effect on the Martensitic transformation. This can be further supported by the fact that similar small-volume $\gamma$-phase to large-volume $\alpha$-phase Martensitic transformations take place in FePt alloys which do not have anti-Invar properties.

We have shown that rest Austenite in $\mathrm{Fe}_{70} \mathrm{Ni}_{30}$ exhibits Invar behavior. This result shows that the properties of the pure Austenite phase are carried into the mixed Austenite-Martensite phase. Invar properties can be observed in this mixed state in a small concentration range around the critical concentration where $T_{C}$ is expected to vanish rapidly with increasing $F e$ concentration.

\section{ACKNOWLEDGEMENTS}

We would like to thank Prof. W. Pepperhoff for continued interest and helpful discussions. This work was supported by the Deutsche Forschungsgemeinschaft (SFB 166).

\section{References}

[1.] Stepakoff G. and Kaufmann L., Acta Metall. 16 (1968) 13.

[2.] Kaufmann L. and Bernstein H., Computer Calculations of Phase Diagrams, (Academic Press, N.Y., London 1970).

[3.] Bendick W. and Pepperhoff W., Acta Metall.30 (1982) 679.

[4.] Acet M., Zähres H., Wassermann E.F. and Pepperhoff W., Phys. Rev. B 49 (1994) 6012.

[5.] Wassermann E.F., Ferromagnetic Materials (North Holland, Amsterdam, 1990) pp. 237.

[6.] Wassermann E.F., J. Magn. Magn. Mater. 100 (1991) 346

[7.] Brown P.J., Capellmann H., Deportes J., Givord D., and Ziebeck K.R.A., J. Magn. Magn. Mater. 30 (1983) 335.

[8] Keune W., Ezawa T., Mecedo W.A.A., Glos U., Schletz K.P., and Kirschbaum U., Physica B 161 (1989).

[9] Acet M., Schneider T., Zähres H., Wassermann E.F., and Pepperhoff W., J. Appl. Phys. 75 (1994) 7015.

[10] Hansen M., Constitution of Binary Alloys (McGraw-Hill, New York, 1958). 